\title{
LAG Rheinland-Pfalz: Fit für die Zukunft
}

Unser kleines Ländchen Rheinland-Pfalz hält uns - die Landesarbeitsgemeinschaft der Lehrerinnen und Lehrer Rheinland-Pfalz e.V. - pflegepolitisch zurzeit recht in Atem.

Zum einen stellen wir uns den Herausforderungen, die sich aktuell aus der Institutionalisierung der Pflegekammer ergeben. Viele unserer Mitglieder stellen sich der Doppelbelastung und kandidieren auf einer der 17 Wählerlisten zur ersten Wahl der Vertreterversammlung. Zum anderen ist es notwendig, die Aufgaben der LAG RLP e.V. neben und mit der Pflegekammer zu definieren. Mit unseren Mitgliedern stimmen wir aktuell die grundsätzlichen Zukunftsaufgaben der LAG RLP e.V. gemeinsam ab. Ein erstes World-Cafe in der letzten Mitgliederversammlung am 26.11.2015 erfasste eine Fülle an Wünschen und Ideen. Als eine zentrale Aufgabe sehen wir nach der Institutionalisierung der Pflegekammer, die Bildung einer Arbeitsgruppe aus LAG Mitgliedern und Pflegekammermitgliedern zu Pflegebildungsfragen.

\section{Schulentwicklung und Schulleitung}

Das Referat Schulentwicklung und Schulleitung im BLGS e.V. lud am 24.11.2015 zur traditionellen Fachkonferenz für Schulleitungen in Gesundheits- und Sozialberufen nach Mainz ein. In der Bildungsakademie Erbacher Hof erhielten die Teilnehmer vom stellvertretenden Vorstandsvorsitzenden des BLGS e.V., Michael Breuckmann, die neuesten Informationen und Entwicklungen auf Bundes- und Länderebene zu Ausbildung und Lehre. Der Schwerpunkt der Tagung war jedoch das Thema "Stärkung der Resilienz im Führungsalltag". Unterstützt und begleitet durch den Resilienztrainer Ulrich Paul Rhein wurde im Rahmen des Seminars folgenden Fragen nachgegangen:

- Was ist Resilienz?

-Wie können Grundlagen der Resilienz im Führungsalltag umgesetzt werden?

- Wie kann jeder Einzelne die acht Faktoren der Resilienz im eigenen Führungsalltag einsetzen?
Das zweite herausragende Thema ist für uns das neue Pflegeberufsgesetz. Wir suchen die verstärkte Zusammenarbeit mit unseren KollegInnen aus der Altenpflege, um uns mit einer Stimme für die Generalistik einzusetzen. Es konnten elf Teilnehmer aus sieben Altenpflegeeinrichtungen zur letzten Fortbildungsveranstaltung begrüßt werden. Tanja Schaller, Schulleiterin der Modellschule „Gemeinsame Pflegeausbildung in der Gesundheits- und Krankenpflege, in der Gesundheits- und Kinderkrankenpflege und in der Altenpflege" der Diakonissen Speyer-Mannheim, erläuterte sehr anschaulich ihre Arbeit und Erfahrungen mit der Modellschule. Ihre Quintessenz: Die Generalistik ist bei uns keine Frage mehr, sie wird gelebt mit guten Erfahrungen.

Sabine Czerwinka

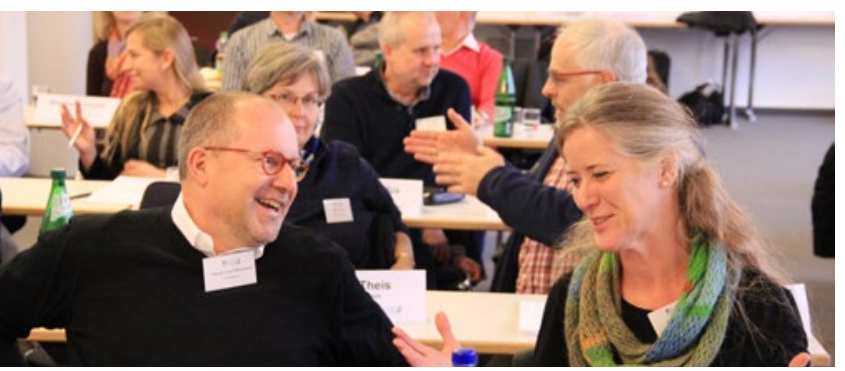

Schulleitungen geben sich gegenseitig Rückmeldung

Eine lebhafte Diskussion beendete die gelungene Veranstaltung. Voraussichtlich wird am 23.11.2016 die nächste Fachkonferenz des Referats Schulentwicklung und Schulleitung im BLGS e.V. in Mainz stattfinden. Nähere Informationen können zeitnah der Homepage www.blgs-ev.de entnommen werden.

Michaela Picker

\section{Veranstaltungen und Termine}

22.-23.01.2016 Kongress Pflege

Ort: Maritim proArte Hotel Berlin www.gesundheitskongresse.de

22.01.2016 BLGS Mitgliederversammlung mit Wahl des Bundesvorstands Ort: Maritim proArte Hotel Berlin www.blgs-ev.de
23.02.2016 LV Berlin

Mitgliederversammlung und Fachtagung

Ort: Wannseeschule Berlin

www.blgs-Iv-berlin.de

10.-12.03.2016 Deutscher Pflegetag

Ort: Station - Berlin

www.deutscher-pflegetag.de

\section{IMPRESSUM}

Herausgeber: Bundesverband Lehrende Gesundheits- und Sozialberufe e.V. (BLGS) Carsten Drude, Vorsitzender (verantwortlich) Alt Moabit 91, 10559 Berlin

Tel.: 030394053 80, Fax: 03039405385 redaktion@blgs-ev.de; www.blgs-ev.de
Der offizielle Newsletter der BLGS erscheint in Kooperation mit HEILBERUFE - Das Pflegemagazin.

Verlag: Springer Medizin Verlag $\mathrm{GmbH}$ Heidelberger Platz 3, 14197 Berlin

Geschäftsführer: Joachim Krieger, Fabian Kaufmann Director Professional Care: Falk H. Miekley

Chefredakteurin: Katja Kupfer-Geißler
Heidelberger Platz 3; 14197 Berlin

Tel.: 03082787 5500, Fax: 030827875505

heilberufe@springer.com

www.springerfachmedien-medizin.de

Layout: Arnulf Illing, Heidelberg

Druck: Phoenix Print GmbH, Würzburg 